\title{
Vinylic Tellurides as Precursors in a Stereoselective and Convergent Route to Z-Enynes and $Z$-Trisubstituted Enediynes
}

\author{
Marcelo A. de Araujo, Cristiano Raminelli and João V. Comasseto*
}

Instituto de Química, Universidade de São Paulo, Av. Prof. Lineu Prestes, 748, 05508-900 São Paulo - SP, Brazil

\begin{abstract}
Teluretos vinílicos $Z$ são transformados em cianocupratos vinílicos $Z$ de ordem superior por transmetalação com dimetil- ou com n-butil(tienil)cianocupratos de lítio. Reação dos cianocupratos vinílicos $Z$ assim obtidos com cloreto de zinco fornece presumivelmente espécies mistas de zinco e cobre que reagem com bromoalquinos levando a eninos e enediinos com retenção da configuração $Z$ da ligação dupla.
\end{abstract}

Vinylic tellurides of $Z$ configuration are transformed into higher order $Z$-vinyl cyanocuprates by transmetallation with higher order lithium dimethyl- or n-butyl(thienyl)cyanocuprates. Reaction of the obtained $Z$-vinyl cyanocuprates with zinc chloride gives presumably mixed zinc-copper species which react with bromoalkynes leading to enynes and enediynes with retention of the $Z$ double bond configuration.

Keywords: Z-Vinylic tellurides, Z-enynes, Z-enediynes, stereoselective synthesis, convergent route

\section{Introduction}

The synthesis of $Z$-vinylic tellurides is now well established. We have developed two methods that can be considered as general routes for the preparation of these compounds (Scheme 1).

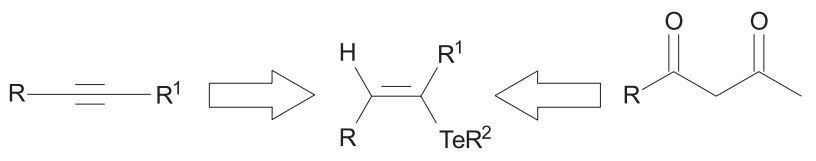

Scheme 1.

The first method uses alkynes ${ }^{1}$ as starting materials and the second method uses $\beta$-dicarbonyl compounds ${ }^{2}$ as precursors. Both classes of compounds are easily accessible. The transformation of vinylic tellurides into higher order vinylic cyano cuprates opened a new perspective for the synthetic application of organotellurium compounds, , $^{1,3}$ since organocopper reagents constitute a key class of organometallic reagents, with numerous applications in organic synthesis. ${ }^{4}$ After 15 years of activity in this field our group has demonstrated that vinylic tellurides are synthetic precursors of higher order Z-vinylic cyanocuprates (Scheme 2).

* e-mail: jvcomass@iq.usp.br

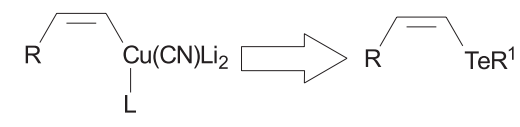

Scheme 2.

Our efforts culminated with the completion of the first total synthesis of a complex molecule making use of an organotellurium precursor. ${ }^{5}$ The first step of this synthesis features the transformation of a $Z$-vinylic telluride into a higher order $Z$-vinylic cyanocuprate.

Some time ago we prepared the first mixed higher order cyano copper-zinc reagent starting from a vinylic telluride and used it to prepare enynes and enediynes systems. ${ }^{6}$ In this paper we give a full account of our findings on the reactivity of this novel organometallic intermediate.

\section{Results and Discussion}

Initially we reacted telluride $\mathbf{1}$ with dilithium butyl(2-thienyl)-cyanocuprate (2) to obtain the dilithium vinyl(2-thienyl)-cyanocuprate (3), which was then reacted for $1 \mathrm{~h}$ at $-50{ }^{\circ} \mathrm{C}$ with haloalkynes $(\mathbf{4 a}, \mathrm{R}=\mathrm{Ph} ; \mathbf{4 b}, \mathrm{R}=$ $\left.n-\mathrm{C}_{5} \mathrm{H}_{11}\right)$. A mixture of the desired enyne $\mathbf{5}$, the dimeric diene 6 and diyne 7 was obtained in a 1:2:1 ratio respectively. By changing the starting dilithium butyl-(2thienyl)-cyanocuprate (2a) for dilithium dimethyl 

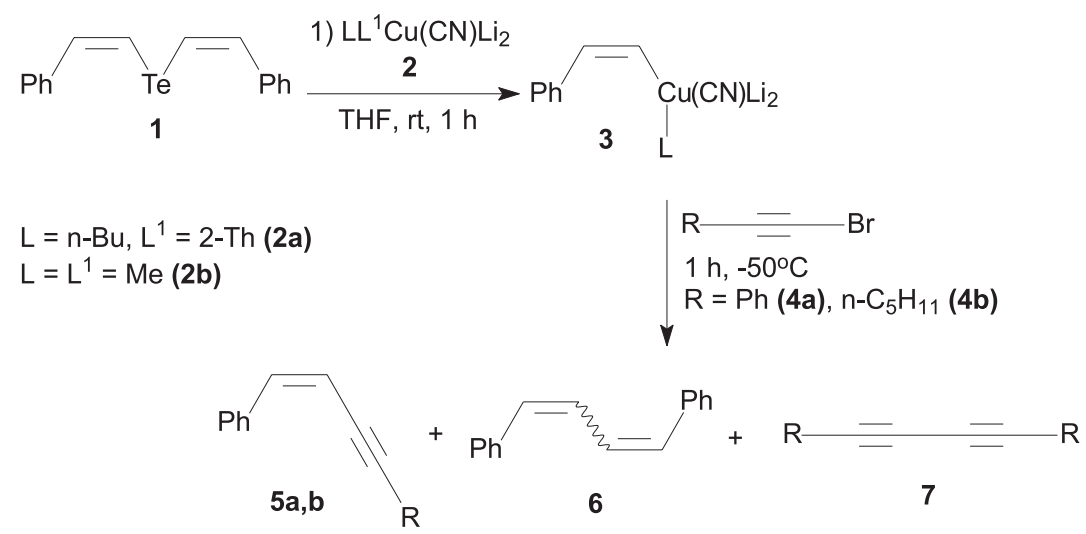

Scheme 3.

cyanocuprate (2b) the results were not improved leading to the same products in a similar ratio (Scheme 3).

Similar results were obtained by Alexakis et al. ${ }^{7}$ who used TMEDA and CuI to circunvent the dimerization problem. This method probably generates a new copper intermediate. Based in this result we applied similar conditions to our systems, as shown in Scheme 4.

$$
\begin{aligned}
& \text { 1) } 2 a \text { or } 2 b, 1 \mathrm{~h}, \mathrm{rt} \\
& \text { 2) } \mathrm{Cul} / \mathrm{TMEDA} \\
& \frac{2.5 \mathrm{~h},-40^{\circ} \mathrm{C}}{\text { 3) } 4 \mathrm{a} \text { or } 4 \mathrm{~b}, 30 \mathrm{~min},-40^{\circ} \mathrm{C}}
\end{aligned} \mathbf{5 , 6 , 7}
$$

\section{Scheme 4.}

Unfortunately the same mixture was formed, this time in a 2:1:1 ration of $\mathbf{5 , 6}$ and $\mathbf{7}$ respectively. Another approach to the synthesis of $\mathbf{5}$ was attempted, by changing the $\mathrm{CuI} /$ TMEDA system for $\mathrm{ZnCl}_{2}\left(1 \mathrm{~mol} \mathrm{~L} \mathrm{~L}^{-1}\right.$ solution in diethyl ether). ${ }^{8}$ The $\mathrm{Zn}$ ions would lead to a mixed cuprate, which could be more selective. However, again a 2:1:1 mixture of 5,6 and 7 was formed. Then we reasoned that the problem could be associated with the nature of the vinyl cuprate. To test this hypothesis we used a different vinylic telluride 8 to generate the vinylic cuprate 9 and then we applied the
$\mathrm{ZnCl}_{2}$ method to perform the coupling reaction, as shown in Scheme 5.

This time the enyne 5a was the only product formed in $85 \%$ isolated yield. In order to test the scope of the coupling reaction, other vinylic tellurides were submitted to the same reaction sequence shown in Scheme 5. The results are depicted in Table 1.

From Table 1 we can observe that vinylic tellurides with the tetrahydropyranyl group at the allylic position give poor yields of the coupling products besides several nonidentified products and thus must be avoided, since the literature reports that this group is prone to react with cuprates leading to ring opening products. ${ }^{4,9}$

With the above results in mind we planned the synthesis of $Z$-enediynes starting from $Z$-vinylic tellurides. $Z$ Enediynes are important fragments present in some antitumoral substances..$^{10}$ In addition, $Z$-enediyne systems can be used in the synthesis of aromatic compounds via a Bergmann cyclization, especially when the reaction is promoted by transition metal complexes. ${ }^{11}$ The advantage of the method proposed by us consists in the easy access to differently substituted $Z$-enediynes by the apropriate combination of the $\mathrm{R}$ group of the vinylic telluride and the $\mathrm{R}^{1}$ group of the bromo-alkyne.

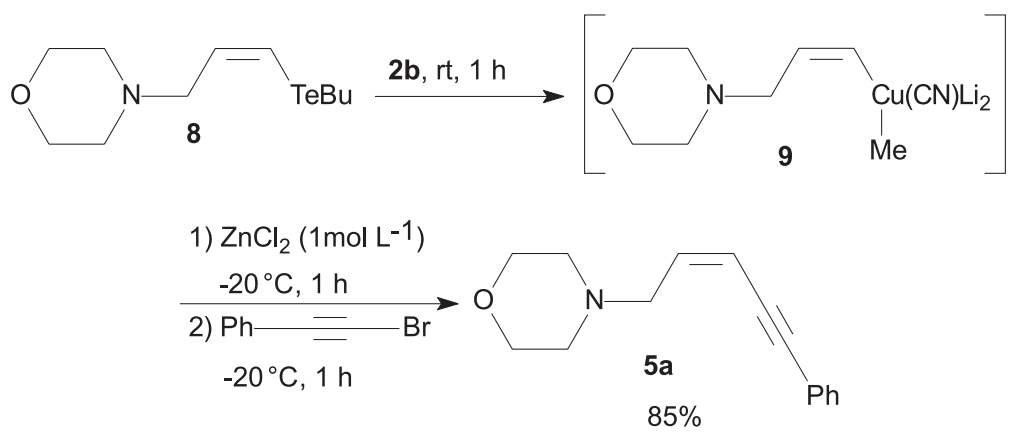

Scheme 5 . 
Table 1. Enynes prepared

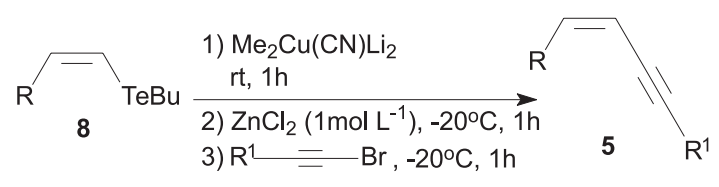

Compound

Isolated yields. Purified by column chromatography; ${ }^{\mathrm{b}} \mathrm{Chromatographic} \mathrm{yield.}$

Application of the above experimental conditions to the coupling of $Z$-telluroenynes and bromoacetylenes led to the $Z$-enediynes in good isolated yields (Table 2).

As can be observed in Table 2, both di- and trisubstituted olefines can be obtained by this method, which constitutes a convergent route to enediynes, presenting advantages over other published methodologies which use multistep processes to introduce different acetylenic moieties. ${ }^{8}$

The $Z$ stereochemistry of the olefinic double bonds of the enynes 5 was easily determined by ${ }^{1} \mathrm{H}$ NMR. The vinylic protons of compound $\mathbf{5 a}$ absorbed as double triplets at $6.02 \mathrm{ppm}(J 10.9,6.7 \mathrm{~Hz})$ and $5.86 \mathrm{ppm}(J 10.9,1.3 \mathrm{~Hz})$; the spectrum of compound $\mathbf{5 d}$ showed double triplets at $5.89 \mathrm{ppm}(J 10.8,6.9 \mathrm{~Hz})$ and at $5.64 \mathrm{ppm}(J 10.8,1.2 \mathrm{~Hz})$. The $Z$ stereochemistry of enediynes $\mathbf{1 0}$ was confirmed by NOE experiments. These spectral data demonstrated that the proposed coupling reaction occurred with retention of the double bond geometry.
The vinylic tellurides used in this work were prepared by reduction of dibutylditelluride ${ }^{1}$ with sodium borohydride in ethanol followed by reaction with the appropriate alkyne (Table 3).

\section{Conclusion}

In conclusion, we developed a highly stereoselective and convergent route to enynes and enediynes. Our method constitutes a straightforward approach to enediynes when compared to the others published in the literature.

\section{Experimental}

\section{Materials}

All solvents used were previously distilled. THF was distilled from sodium/benzophenone under $\mathrm{N}_{2}$ immediately before use. ${ }^{12}$ All organolithium reagents were 
Table 2. Enediynes prepared

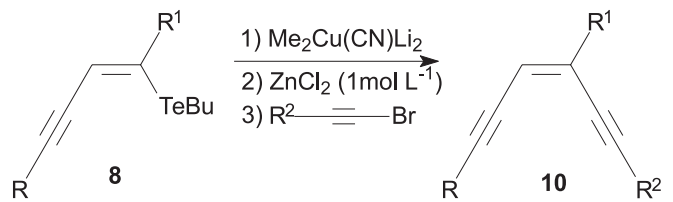

\begin{tabular}{|c|c|c|c|c|c|}
\hline Compound & $\mathrm{R}$ & $\mathrm{R}^{1}$ & $\mathrm{R}^{2}$ & $\begin{array}{l}\text { Yield }^{\mathrm{a}} \\
(\%)\end{array}$ & $\begin{array}{c}\text { Eluente } \\
\text { (hexane:ethyl acetate:methanol) }\end{array}$ \\
\hline $10 a$ & $\mathrm{Ph}$ & $\mathrm{Ph}$ & $\mathrm{Ph}$ & 60 & $97: 3: 0$ \\
\hline $10 \mathrm{~b}$ & $\mathrm{Ph}$ & $\mathrm{Ph}$ & $\mathrm{C}_{5} \mathrm{H}_{11}$ & 55 & $98: 2: 0$ \\
\hline $10 \mathrm{c}$ & $\mathrm{Me}$ & $\mathrm{Me}$ & $\mathrm{Ph}$ & 73 & $97: 3: 0$ \\
\hline 10d & $\mathrm{Me}$ & $\mathrm{Me}$ & $\mathrm{C}_{5} \mathrm{H}_{11}$ & 55 & $98: 2: 0$ \\
\hline $10 \mathrm{e}$ & & & $\mathrm{Ph}$ & $66^{\mathrm{b}}$ & $0: 95: 5$ \\
\hline $10 \mathrm{f}$ & & & $\mathrm{C}_{5} \mathrm{H}_{11}$ & 55 & $0: 95: 5$ \\
\hline $10 \mathrm{~g}$ & $\mathrm{C}_{4} \mathrm{H}_{9}$ & $\mathrm{H}$ & $\mathrm{Ph}$ & 80 & 100:0:0 \\
\hline $10 \mathrm{~h}$ & $\mathrm{C}_{4} \mathrm{H}_{9}$ & $\mathrm{H}$ & $\mathrm{C}_{5} \mathrm{H}_{11}$ & 78 & 100:0:0 \\
\hline
\end{tabular}

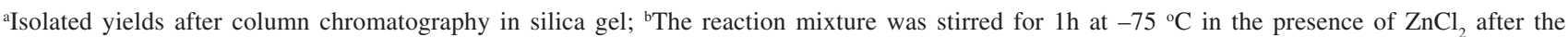
transmetallation and $1 \mathrm{~h}$ at the same temperature after the addition of the bromoalkyne. The reaction was performed in a $4 \mathrm{mmol}$ scale.

Table 3. Butylvinyltellurides prepared

$$
\mathrm{R}=\mathrm{R}^{1} \stackrel{(\mathrm{BuTe})_{2}, \mathrm{NaBH}_{4}, \mathrm{EtOH},}{\mathrm{N}_{2} \text {, reflux }} \underset{\mathrm{R}}{-} \overbrace{\mathrm{TeBu}}^{\mathrm{R}^{1}}
$$

\begin{tabular}{|c|c|c|c|c|c|}
\hline Compound & $\mathrm{R}$ & $\mathrm{R}^{1}$ & $\begin{array}{l}\text { Reaction time } \\
\text { (h) }\end{array}$ & $\begin{array}{l}\text { Yield } \\
(\%)\end{array}$ & $\begin{array}{c}\text { Eluent } \\
\text { (hexane:ethyl acetate) }\end{array}$ \\
\hline $8 \mathbf{a}$ & & $\mathrm{H}$ & 2.5 & 75 & $93: 7$ \\
\hline $8 \mathbf{b}$ & & $\mathrm{H}$ & 3.0 & 73 & $95: 5$ \\
\hline $8 \mathrm{c}$ & & $\mathrm{H}$ & 3.0 & 78 & $95: 5$ \\
\hline $8 d$ & & $\mathrm{Ph}$ & 3.0 & 80 & $100: 0$ \\
\hline $8 e$ & & $\mathrm{Me}$ & 2.5 & 92 & $100: 0$ \\
\hline $8 f$ & & & 4.0 & 89 & $50: 50$ \\
\hline $8 \mathrm{~g}$ & & $\mathrm{H}$ & 1.0 & 83 & $100: 0$ \\
\hline
\end{tabular}


titrated using 1,10-phenanthroline as indicator prior to use. $^{13}$ Tellurium metal (200 mesh) was obtained from Aldrich Chemical Co. and dried overnight in an oven at $100{ }^{\circ} \mathrm{C}$. $\mathrm{CuCN}$ was purchased from the Aldrich Chemical Co. and then dried under $\mathrm{P}_{2} \mathrm{O}_{5}$ and vacuum in an Abderhalden apparatus overnight and then stored in a stoppered flask. Column chromatographic separations were carried out with Merck or Aldrich silica gel (230-400 mesh). 2-Prop-2-ynyloxy-tetrahydro-pyran, ${ }^{14}$ 2-pent-2-en4-ynyloxy-tetrahydro-pyran, ${ }^{14}$ 4-prop-2-ynylmorpholine, ${ }^{15}$ 4,4'-(hexa-2,4-diynyl)-bismorpholine, ${ }^{15}$ bromoethynyl-benzene ${ }^{15}$ and 1-bromo heptyne ${ }^{15}$ were prepared according to literature procedure. The remaining chemicals were obtained from commercial sources. All reactions were carried out under an inert atmosphere of dry and deoxygenated [by bubbing through a solution of $\mathrm{VCl}_{3}$ (pH 1) with $\mathrm{Zn} / \mathrm{Hg}$ amalgam] $\mathrm{N}_{2}$. All operations were carried out in flame-dried glassware. ${ }^{1} \mathrm{H}$ spectra were recorded on a $200 \mathrm{MHz}$ and ${ }^{13} \mathrm{C} \mathrm{NMR}$ at $50 \mathrm{MHz}$ spectrometers, except where noted otherwise. All spectra were taken in $\mathrm{CDCl}_{3}$ with tetramethylsilane (TMS) as internal standard. Chemical shifts are given in ppm downfield of the reference. Mass spectra were obtained on a MS-HP 5890/5988 AE1 902 Vg micromass 70E and on a FINIGAN INCOS-50 instruments. Elemental analyses were performed at the Microanalytical Laboratory of the Institute of Chemistry, Universidade de São Paulo. The IUPAC names were obtained using the software ISIS ${ }^{\mathrm{TM}}$ / Draw, version 2.4.

\section{Preparation of dibutyl ditelluride ${ }^{1}$}

To a suspension of Te (Aldrich $200 \mathrm{mesh}, 40 \mathrm{mmol}$, $5,10 \mathrm{~g})$ in THF $(50 \mathrm{~mL})$ under nitrogen at $0{ }^{\circ} \mathrm{C}$ was added $n$-BuLi (44 mmol, $22 \mathrm{~mL}$ of a solution $2.0 \mathrm{~mol} \mathrm{~L}^{-1}$ in hexane). The mixture was stirred for $35 \mathrm{~min}$ at $0{ }^{\circ} \mathrm{C}$ and for additional $15 \mathrm{~min}$ at room temperature. The mixture was cautiously poured into an Erlenmeyer flask containing water $(100 \mathrm{~mL})$ and stirred in presence of atmospheric oxygen for $15 \mathrm{~min}$. The organic phase was then separated, diluted with ethyl acetate $(100 \mathrm{~mL})$, washed with saturated solution of $\mathrm{NH}_{4} \mathrm{Cl}(200 \mathrm{~mL})$ and brine $(200 \mathrm{~mL})$, and dried with $\mathrm{MgSO}_{4}$. Dibutyl ditelluride was obtained after filtration and removal of the solvent. Yield $6.79 \mathrm{~g}$ $(92 \%)$.

\section{Preparation of the vinylic tellurides ${ }^{1}$}

To a solution of dibutyl ditelluride (5.54 g, $15 \mathrm{mmol}$ ) and the appropriate alkyne $(33 \mathrm{mmol})$ in absolute ethanol $(90 \mathrm{~mL})$ under deoxygenated nitrogen and magnetic stirring was added $\mathrm{NaBH}_{4}(1.6 \mathrm{~g}, 42 \mathrm{mmol})$ in small portions until the color of the solution turned from dark red to pale yellow. The mixture was then refluxed for the time indicated in Table 3. The mixture was cooled to room temperature and treated with water $(15 \mathrm{~mL}), 10 \%$ aqueous sodium hydroxide $(15 \mathrm{~mL})$ and ethyl acetate $(600 \mathrm{~mL})$. The organic phase was separated and washed with water $(2 \times 300 \mathrm{~mL})$ and with saturated $\mathrm{NaCl}$ solution $(2 \times 300 \mathrm{~mL})$. After drying $\left(\mathrm{MgSO}_{4}\right)$ and filtration, the solvent was evaporated under reduced pressure, and the residue was purified by flash chromatography on silica gel using the appropriate eluent (Table 3).

4-(3-Butyltellanyl-(Z)-allyl)-morpholine (8a). ${ }^{16}$ Yield: 6.99g (75\%); ${ }^{1} \mathrm{H} \mathrm{NMR}\left(\mathrm{CDCl}_{3}\right) \delta 6.90(\mathrm{~d}, J 9.6 \mathrm{~Hz}, 1 \mathrm{H})$, $6.34(\mathrm{dd}, J 9.6 \mathrm{~Hz}, 5.1 \mathrm{~Hz}, 1 \mathrm{H}), 3.72$ (t, J 5.0 Hz, 4H), 2.91 (d, J 5.1 Hz, 2H), 2.51-2.42 (m, 6H), 1.76 (quint, $J 7.1 \mathrm{~Hz}$, 2H), 1.38 (sext, J 7.1 Hz, 2H), 0.91 (t, J 7.2 Hz, 3H); ${ }^{13} \mathrm{C}$ NMR $\left(\mathrm{CDCl}_{3}\right) \delta 132.1,108.5,66.7,60.5,53.0,33.9,24.9$, $13.3,7.5$.

2-(3-Butyltellanyl-(Z)-allyloxy)-tetrahydro-pyran (8b). ${ }^{16}$ Yield: $7.14 \mathrm{~g}(73 \%) ;{ }^{1} \mathrm{H}$ NMR $\left(\mathrm{CDCl}_{3}\right) \delta 6.83$ (dt, $J 9.9 \mathrm{~Hz}, 1.3 \mathrm{~Hz}, 1 \mathrm{H}), 6.40$ (dt, J9.9 Hz, $5.6 \mathrm{~Hz}, 1 \mathrm{H}), 4.66-$ $4.63(\mathrm{~m}, 1 \mathrm{H}), 4.00$ (dd, J 5.6 Hz, $1.3 \mathrm{~Hz}, 2 \mathrm{H}), 3.56-3.53(\mathrm{~m}$, 2H), 2.63 (t, J 7.2 Hz, 2H), 1.85-1.50 (m, 8H), 1.43 (sext, $J 7.2 \mathrm{~Hz}, 2 \mathrm{H}), 0.91(\mathrm{t}, J 7.2 \mathrm{~Hz}, 3 \mathrm{H}) ;{ }^{13} \mathrm{C} \mathrm{NMR}\left(\mathrm{CDCl}_{3}\right) \delta$ 134.7, 105.7, 98.0, 87.2, 73.7, 68.5, 61.9, 34.0, 30.4, 25.1, $19.2,13.3,7.0$.

2-(5-Butyltellanyl-penta-(2E,4Z)-dienyloxy)tetrahydro-pyran $(\mathbf{8 c}) .{ }^{1}$ Yield: $8.23 \mathrm{~g}(78 \%) ; 360 \mathrm{MHz}$ ${ }^{1} \mathrm{H} \mathrm{NMR}\left(\mathrm{CDCl}_{3}\right) \delta$ 6.81-6.77 (m, 2H), 6.25 (ddt, $J 15.1 \mathrm{~Hz}$, $9.3 \mathrm{~Hz}, 1.4 \mathrm{~Hz}, 1 \mathrm{H}), 5.88$ (dt, J 15.1 Hz, 6.3 Hz, 1H), 4.66 (t, J $3.5 \mathrm{~Hz}, 1 \mathrm{H}), 4.29$ (ddd, J $13.2 \mathrm{~Hz}, 5.6 \mathrm{~Hz}, 1.5 \mathrm{~Hz}, 1 \mathrm{H}$ ), 4.05 (ddd, $J 13.2 \mathrm{~Hz}, 6.6 \mathrm{~Hz}, 1.3 \mathrm{~Hz}, 1 \mathrm{H}), 3.90-3.81$ (m, 1H), 3.55-3.49 (m, 1H), $2.71(\mathrm{t}, J 7.5 \mathrm{~Hz}, 2 \mathrm{H}), 0.92(\mathrm{t}, J 7.5$ $\mathrm{Hz}, 3 \mathrm{H}) ; 90 \mathrm{MHz}{ }^{13} \mathrm{C} \mathrm{NMR}\left(\mathrm{CDCl}_{3}\right) \delta 136.84,132.93$, 131.32, 106.03, 97.75, 67.09, 61.99, 33.98, 30.47, 25.32, 24.78, 19.27, 13.28, 6.82 .

1-Butyltellanyl-1,4-diphenyl-but-(1Z)-en-3-ynyl (8d). ${ }^{1}$ Yield: 9.28g (80\%); ${ }^{1} \mathrm{H} \mathrm{NMR}\left(\mathrm{CDCl}_{3}\right) \delta 7.66-7.32(\mathrm{~m}, 10 \mathrm{H})$, $6.46(\mathrm{~s}, 1 \mathrm{H}), 2.60$ (t, $J 7.5 \mathrm{~Hz}, 2 \mathrm{H}), 1.68$ (quint, $J 7.5 \mathrm{~Hz}$, 2H), 1.30 (sext, J 7.5 Hz, 2H), 0.85 (t, J 7.5 Hz, 3H); ${ }^{13} \mathrm{C}$ NMR $\left(\mathrm{CDCl}_{3}\right) \delta 142.1,137.1,131.3,128.3,128.0,123.3$, 116.2, 90.4, 33.8, 24.9, 13.2, 8.9; LRMS m/z (relative intensity) 390 (14), 334 (3), 231 (2), 202 (100), 145 (5), 126 (5), 77 (7).

2-Butyltellanyl-hex-(2Z)-en-4-yne (8e). ${ }^{1}$ Yield: $7.28 \mathrm{~g}$ $(92 \%) ;{ }^{1} \mathrm{H} \mathrm{NMR}\left(\mathrm{CDCl}_{3}\right) \delta$ 5.50-5.91 (m, 1H), 2.78 (t, J 7.4 $\mathrm{Hz}, 2 \mathrm{H}), 2.21$ (m, 3H), 1.99 (d, J 2.6 Hz, 3.0 Hz, 3H), 1.78 (quint, J7.1 Hz, 2H), 1.40 (sext, J7.4 Hz, 2H), 0.92 (t, J 7.2 $\mathrm{Hz}, 3 \mathrm{H}) ;{ }^{13} \mathrm{C} \mathrm{NMR}\left(\mathrm{CDCl}_{3}\right) \delta 128.7,114.2,91.1,79.6,34.4$, 26.9, 25.1, 13.4, 4.8, 4.4; LRMS $m / z$ (relative intensity) 
266 (24), 207 (6), 167 (2), 143 (2), 91 (5), 80 (100), 63 (7), 57 (26).

4,4'-(2-Butyltellanyl-hex-(2Z)-en-4-ynyl)bismorpholine (8f). Yield: $11.56 \mathrm{~g}(89 \%) ;{ }^{1} \mathrm{H} \mathrm{NMR}\left(\mathrm{CDCl}_{3}\right)$ $\delta 6.27(\mathrm{~s}, 1 \mathrm{H}), 3.66-3.76(\mathrm{~m}, 8 \mathrm{H}), 3.46(\mathrm{~d}, J 1.8 \mathrm{~Hz}, 2 \mathrm{H})$, $3.21(\mathrm{~s}, 2 \mathrm{H}), 2.98(\mathrm{t}, J 7.5 \mathrm{~Hz}, 2 \mathrm{H}), 2.61(\mathrm{t}, J 4.5 \mathrm{~Hz}, 4 \mathrm{H})$, $2.42(\mathrm{t}, J 4.5 \mathrm{~Hz}, 4 \mathrm{H}$ ), 1.79 (quint, $J 7.3 \mathrm{~Hz}, 2 \mathrm{H}$ ), 1.38 (sext, $J 7.3 \mathrm{~Hz}, 2 \mathrm{H}), 0.92(\mathrm{t}, J 7.2 \mathrm{~Hz}, 3 \mathrm{H}) ;{ }^{13} \mathrm{C} \mathrm{NMR}\left(\mathrm{CDCl}_{3}\right) \delta$ 133.2, 115.2, 90.3, 84.4, 67.5, 66.8, 66.6, 53.0, 52.1, 47.9, 34.1, 25.0, 13.3, 5.5; LRMS $\mathrm{m} / z$ (relative intensity) 436 (2), 377 (1), 349 (1), 292 (18), 249 (32), 164 (51), 100 (100), 86 (21), 77 (38), 56 (29); Elemental Analysis Calcd. for $\mathrm{C}_{18} \mathrm{H}_{30} \mathrm{~N}_{2} \mathrm{O}_{2} \mathrm{Te} \mathrm{C}, 49.81, \mathrm{H}, 6.97, \mathrm{~N}, 6.45$; Found C, 49,72, $\mathrm{H}, 6,81, \mathrm{~N}, 6,27 \%$.

1-Butyltellanyl-oct-(1Z)-en-3-yne (8g). Yield: $7.27 \mathrm{~g}$ (83\%); ${ }^{1} \mathrm{H} \mathrm{NMR}\left(\mathrm{CDCl}_{3}\right) \delta 7.16(\mathrm{~d}, J 10.3 \mathrm{~Hz}, 1 \mathrm{H}), 6.36(\mathrm{dt}$, $J 10.3 \mathrm{~Hz}, 2.2 \mathrm{~Hz}, 1 \mathrm{H}), 2.74(\mathrm{t}, J 7.4 \mathrm{~Hz}, 2 \mathrm{H}), 2.36(\mathrm{td}, J 7.3$ $\mathrm{Hz}, 2.2 \mathrm{~Hz}, 2 \mathrm{H}$ ), 1.81 (quint, $J 7.3 \mathrm{~Hz}, 2 \mathrm{H}$ ), 1.31-1.58 (m, $6 \mathrm{H}), 0.92(\mathrm{t}, J 7.3 \mathrm{~Hz}, 6 \mathrm{H}) ;{ }^{13} \mathrm{C} \mathrm{NMR}\left(\mathrm{CDCl}_{3}\right) \delta 118.0$, 117.9, 97.9, 80.8, 34.4, 24.9, 22.0, 19.3, 13.6, 13.4; LRMS $\mathrm{m} / \mathrm{z}$ (relative intensity) 294 (46), 237 (11), 233 (6), 195 (100), 107 (46), 79 (56), 57 (20); Elemental Analysis Calcd. for $\mathrm{C}_{12} \mathrm{H}_{20} \mathrm{Te} \mathrm{C}, 48.97, \mathrm{H}, 6.85$; Found C, 49,15, H, 6,59\%.

\section{Preparation of the enynes}

To a suspension of $\mathrm{CuCN}(0.09 \mathrm{~g}, 1 \mathrm{mmol})$ in THF $(5 \mathrm{~mL})$ under magnetic stirring and nitrogen atmosphere, at $-75^{\circ} \mathrm{C}$, was added methyllithium $(1.67 \mathrm{~mL}, 2.0 \mathrm{mmol}$ of a $1.2 \mathrm{~mol} \mathrm{~L}^{-1}$ solution in ether). The homogeneous solution was stirred for $15 \mathrm{~min}$ at $-20^{\circ} \mathrm{C}$ and then heated to room temperature. The appropriate vinylic telluride $(1.1 \mathrm{mmol})$ in THF $(2 \mathrm{~mL})$ was then added and the mixture was stirred at room temperature for $1 \mathrm{~h}$ and then cooled to $-20^{\circ} \mathrm{C}$. After that, $\mathrm{ZnCl}_{2}\left(1.0 \mathrm{~mL}, 1.0 \mathrm{mmol}\right.$ of a $1 \mathrm{~mol} \mathrm{~L}^{-1}$ solution in diethyl ether) was added and the mixture was stirred for $1 \mathrm{~h}$. The appropriate bromoalkyne $(1.1 \mathrm{mmol})$ in THF $(1 \mathrm{~mL})$ was added and the mixture was stirred for $1 \mathrm{~h}$ at the same temperature. A mixture (4:1) of saturated solutions of ammonium chloride and ammonium hydroxide was added $(40 \mathrm{~mL})$ and the reaction was extracted with ethyl acetate $(3 \times 30 \mathrm{~mL})$. The combined organic phases were washed with brine $(2 \times 50 \mathrm{~mL})$, dried over magnesium sulfate and filtered. The organic solvent was evaporated under reduced pressure, and the residue was purified by column chromatography on silica gel, eluting first with hexane and then with hexane/ethyl acetate (Table 1).

4-(5-Phenyl-pent-(2Z)-en-4-ynyl)-morpholine (5a). Yield: $0.193 \mathrm{~g}(85 \%) ;{ }^{1} \mathrm{H}$ NMR $\left(\mathrm{CDCl}_{3}\right) \delta 7.46-7.29(\mathrm{~m}$, $5 \mathrm{H}), 6.02(\mathrm{dt}, J 10.9 \mathrm{~Hz}, J 6.7 \mathrm{~Hz}, 1 \mathrm{H}), 5.86(\mathrm{dt}, J 10.9 \mathrm{~Hz}$,
$J 1.3 \mathrm{~Hz} 1 \mathrm{H}), 3.71(\mathrm{~m}, 4 \mathrm{H}), 3.32(\mathrm{~d}, J 4.0 \mathrm{~Hz}, 2 \mathrm{H}), 2.51$ (m, $4 \mathrm{H}) ;{ }^{13} \mathrm{C} \mathrm{NMR}\left(\mathrm{CDCl}_{3}\right) \delta 138.7,131.2,128.2,123.0,112.3$, 94.7, 85.4, 66.8, 57.7, 53.51; LRMS $\mathrm{m} / \mathrm{z}$ (relative intensity) 226 (26), 21 (15), 198 (12), 182 (56), 171 (12), 168 (83), 156 (23), 150 (48), 142 (27), 141 (100), 139 (28), 91 (14), 56 (14); Elemental Analysis Calcd. for $\mathrm{C}_{15} \mathrm{H}_{17} \mathrm{NO}$ C, 79.23, H, 7.48, N, 6.27; Found C, 78.36, H, 7.69, N, 5.90\%.

4-(Dec-(2Z)-en-4-ynyl)-morpholine (5d). Yield: 0.144g (65\%); ${ }^{1} \mathrm{H} \mathrm{NMR}\left(\mathrm{CDCl}_{3}\right) \delta 5.89(\mathrm{dt}, J 10.8 \mathrm{~Hz}, 6.9 \mathrm{~Hz}, 1 \mathrm{H})$, $5.64(\mathrm{dt}, J 10.8 \mathrm{~Hz}, 1.2 \mathrm{~Hz}, 1 \mathrm{H}), 3.72(\mathrm{t}, J 4.6 \mathrm{~Hz}, 4 \mathrm{H}), 3.25$ $(\mathrm{dd}, J 6.9 \mathrm{~Hz}, 1.2 \mathrm{~Hz}, 2 \mathrm{H}), 2.49(\mathrm{t}, J 4.6 \mathrm{~Hz}, 4 \mathrm{H}) ;{ }^{13} \mathrm{C} \mathrm{NMR}$ $\left(\mathrm{CDCl}_{3}\right) \delta 137.1,113.0,96.2,92.2,66.9,57.6,53.5,31.0$, 28.4, 22.1, 19.4, 14.0; LRMS $\mathrm{m} / \mathrm{z}$ (relative intensity) 220 (4), 164 (100), 120 (64), 91 (32), 77 (45), 56 (35); Elemental Analysis Calcd. for $\mathrm{C}_{14} \mathrm{H}_{23} \mathrm{NO}$ C, 75.97, H, 10.47, N, 6.33; Found C, 75.62, H, 10.48, N, 6.10\%.

\section{Preparation of the enediynes}

Dilithium dimethylcyanocuprate $(1 \mathrm{mmol})$ was prepared as described above. To this homogeneous solution was added the appropriate vinylic telluride $(1.1 \mathrm{mmol})$ in THF $(2 \mathrm{~mL})$ at room temperature. The mixture was stirred for $1 \mathrm{~h}$ at the same temperature and then cooled to $-20^{\circ} \mathrm{C}$. After that, $\mathrm{ZnCl}_{2}\left(1.0 \mathrm{~mL}, 1.0 \mathrm{mmol}\right.$ of a $1 \mathrm{~mol} \mathrm{~L}^{-1}$ solution in diethyl ether) was added and the mixture was stirred for $1.5 \mathrm{~h}$. The appropriate bromoalkyne $(1.1 \mathrm{mmol})$ in THF $(1 \mathrm{~mL})$ was added and the mixture was stirred for $1 \mathrm{~h}$ at the same temperature. A mixture (4:1) of saturated solutions of ammonium chloride and ammonium hydroxide was added $(40 \mathrm{~mL})$ and the reaction was extracted with ethyl acetate $(3 \times 30 \mathrm{~mL})$. The combined organic phases were washed with brine $(2 \times 50 \mathrm{~mL})$, dried over magnesium sulfate and filtered. The organic solvent was evaporated under reduced pressure, and the residue was purified by column chromatography on silica gel, eluting first with hexane and then with hexane/ethyl acetate/methanol (Table 2).

1,3,6-Triphenyl-hex-(3Z)-en-1,5-diyne (10a). Yield: $0.182 \mathrm{~g}(60 \%)$; ${ }^{1} \mathrm{H}$ NMR $\left(\mathrm{CDCl}_{3}\right) \delta$ 7.76-7.71 (m, 2H), 7.63$7.50(\mathrm{~m}, 4 \mathrm{H}), 7.40-7.31(\mathrm{~m}, 9 \mathrm{H}), 6.60(\mathrm{~s}, 1 \mathrm{H}) ;{ }^{13} \mathrm{C} \mathrm{NMR}$ $\left(\mathrm{CDCl}_{3}\right) \delta 136.7,133.4,131.7,131.6,128.8,128.7,128.6$, 128.5, 128.4, 128.0, 126.1, 123.44, 123.11, 113.7, 98.5, 89.0, 87.6; LRMS m/z (relative intensity) 304 (100), 226 (9), 202 (7), 178 (6), 150 (10), 77 (2); Elemental Analysis Calcd. for $\mathrm{C}_{24} \mathrm{H}_{16} \mathrm{C}, 94.70, \mathrm{H}, 5.30$; Found C, 94.43, H, $5.47 \%$.

1,4-Diphenyl-undec-(3Z)-en-1,5-diyne (10b). Yield: $0.164 \mathrm{~g}(55 \%)$; ${ }^{1} \mathrm{H} \mathrm{NMR}\left(\mathrm{CDCl}_{3}\right)$ d 7.69-7.64 (m, 2H), 7.527.47 (m, 2H), 7.38-7.30 (m, 6H), $6.46(\mathrm{~s}, 1 \mathrm{H}), 2.55$ (t, J 7.2 $\mathrm{Hz}, 2 \mathrm{H}$ ), 1.68 (quint, $J 7.2 \mathrm{~Hz}, 2 \mathrm{H}) 1.56-1.28(\mathrm{~m}, 4 \mathrm{H}) 0.87$ 
(t, J 7.2 Hz, 3H); ${ }^{13} \mathrm{C} \mathrm{NMR}\left(\mathrm{CDCl}_{3}\right) \delta 131.7,128.6,128.4$, 128.3, 126.1, 112.5, 31.1, 28.5, 22.2, 19.9, 13.9; LRMS $\mathrm{m} / \mathrm{z}$ (relative intensity) 298 (100), 239 (76), 226 (19), 215 (18), 165 (28), 115 (31), 91 (21); Elemental Analysis Calcd. for $\mathrm{C}_{23} \mathrm{H}_{22} \mathrm{C}, 92.57, \mathrm{H}, 7.43$; Found C, 92.41, H, 7.53\%.

3-Methyl-1-phenyl-hept-(3Z)-en-1,5-diyne (10c). Yield: $0.132 \mathrm{~g}(73 \%)$; ${ }^{1} \mathrm{H} \mathrm{NMR}\left(\mathrm{CDCl}_{3}\right) \delta 7.50-7.45$ (m, 2H), 7.33 $7.29(\mathrm{~m}, 3 \mathrm{H}), 5.68-5.66(\mathrm{~m}, 1 \mathrm{H}), 2.05$ (d, J $2.3 \mathrm{~Hz}, 3 \mathrm{H}), 1.99$ $(\mathrm{s}, 3 \mathrm{H}) ;{ }^{13} \mathrm{C} \mathrm{NMR}\left(\mathrm{CDCl}_{3}\right) \delta 131.6,128.9,128.2,127.2$, 123.2, 115.8, 94.9, 91.5, 89.2, 77.9, 22.7, 4.6; LRMS m/z (relative intensity) 180 (100), 165 (54), 152 (11), 139 (12), 126 (13), 115 (10), 89 (15), 76 (15), 63 (12); Elemental Analysis Calcd. for $\mathrm{C}_{14} \mathrm{H}_{12} \mathrm{C}, 93.29, \mathrm{H}, 6.71$; Found $\mathrm{C}$, 92.89, H, 6.83\%.

5-Methyl-dodec-(4Z)-en-2,6-diyne (10d). Yield: 0.096g (55\%); ${ }^{1} \mathrm{H} \mathrm{NMR}\left(\mathrm{CDCl}_{3}\right) \delta$ 5.54-5.52 (m, 1H), 2.40 (t, J 6.8 $\mathrm{Hz}, 2 \mathrm{H}), 2.00$ (d, $J 1.8 \mathrm{~Hz}, 3 \mathrm{H}) 1.61-1.28$ (m, 6H), 1.88 (d, $J$ $0.73 \mathrm{~Hz}, 3 \mathrm{H}), 0.91(\mathrm{t}, J 6.8 \mathrm{~Hz}, 3 \mathrm{H}) ;{ }^{13} \mathrm{C} \mathrm{NMR}\left(\mathrm{CDCl}_{3}\right) \delta$ 129.8, 114.1, 96.7, 90.0, 80.5, 77.9, 30.9, 28.3, 23.3, 22.2, 19.6, 14.0, 4.6; LRMS $m / z$ (relative intensity) 174 (75), 145 (28), 129 (41), 115 (93), 105 (40), 91 (100), 77 (34), 65 (21); Elemental Analysis Calcd. for $\mathrm{C}_{13} \mathrm{H}_{18} \mathrm{C}, 89.59, \mathrm{H}$, 10.41; Found C, 89.84, H, 10.81\%.

4,4'-(2-Phenylacetylenyl-hex-(2Z)-en-4-ynyl)bismorpholine (10e). Yield: 0.231g (66\%); ${ }^{1} \mathrm{H}$ NMR $\left(\mathrm{CDCl}_{3}\right) \delta$ 7.52-7.27 (m, 5H), $6.01(\mathrm{~m}, 1 \mathrm{H}), 3.75-3.64(\mathrm{~m}$, $8 \mathrm{H}), 3.51$ (d, J 2.0 Hz, 2H), 3.17 (d, J1.3 Hz, 2H) 2.61 (t, $J$ $4.7 \mathrm{~Hz}, 4 \mathrm{H}), 2.53(\mathrm{t}, J 4.6 \mathrm{~Hz}, 4 \mathrm{H}) ;{ }^{13} \mathrm{C} \mathrm{NMR}\left(\mathrm{CDCl}_{3}\right) \delta$ 159.1, 131.7, 131.2, 128.6, 128.3, 122.9, 116.2, 91.4, 88.1, 83.4, 67.0, 66.8, 62.9, 53.3, 52.1, 48.3; LRMS $m / z$ (relative intensity) 349 (7), 264 (29), 234 (8), 206 (16), 191 (8), 178 (34), 165 (24), 100 (100), 86 (7), 77 (5), 56 (24); Elemental Analysis Calcd. for $\mathrm{C}_{22} \mathrm{H}_{26} \mathrm{~N}_{2} \mathrm{O}_{2} \mathrm{C}, 75.40, \mathrm{H}, 7.48, \mathrm{~N}, 7.90$; Found C, 75.59, H, 7.45, N, 8.01\%.

4,4'-(2-heptynyl-hex-(2Z)-en-4-ynyl)-bismorpholine (10f). Yield: 0.190g (55\%); ${ }^{1} \mathrm{H}$ NMR $\left(\mathrm{CDCl}_{3}\right) \delta 5.87(\mathrm{t}, J$ $1.4 \mathrm{~Hz}, 1 \mathrm{H}), 3.77-3.68$ (m, 8H), 3.49 (d, J $2.0 \mathrm{~Hz}, 2 \mathrm{H}) 3.04$ (d, J 1.2 Hz, 2H), 2.63 (t, J 4.7 Hz, 4H), 2.47 (t, J 4.7 Hz, $4 \mathrm{H}), 2.39$ (t, J 7.0 Hz, 2H), 1.61-1.25 (m, 6H), 0.90 (t, J 6.9 $\mathrm{Hz}, 3 \mathrm{H}) ;{ }^{13} \mathrm{C} \mathrm{NMR}\left(\mathrm{CDCl}_{3}\right) \delta 131.9,115.0,114.8,98.5$, 89.8, 83.5, 79.3, 66.9, 66.8, 63.4, 53.3, 52.1, 48.2, 31.0, $28.3,22.2,19.7,14.0 ;$ LRMS $m / z$ (relative intensity) 344 (4), 257 (43), 228 (11), 214 (6), 173 (7), 156 (6), 143 (7), 129 (9), 115 (15), 100 (100), 86 (9), 77 (9), 55 (10); Elemental Analysis Calcd. for $\mathrm{C}_{21} \mathrm{H}_{32} \mathrm{~N}_{2} \mathrm{O}_{2} \mathrm{C}, 73.22$, $\mathrm{H}, 9.36$, N, 8.13; Found C, 72.86, H, 9.46, N, 8.30\%.

Dec-(3Z)-ene-1,5-diynyl-benzene (10g). Yield: $0.166 \mathrm{~g}$ $(80 \%) ;{ }^{1} \mathrm{H}$ NMR $\left(\mathrm{CDCl}_{3}\right) \delta$ 7.49-7.44 (m, 2H), 7.32-7.24 $(\mathrm{m}, 3 \mathrm{H}), 5.96(\mathrm{~d}, J 10.8 \mathrm{~Hz}, 1 \mathrm{H}), 5.85(\mathrm{dt}, J 10.7 \mathrm{~Hz}, 1.9 \mathrm{~Hz}$, 1H) 2.44 (dt, J 6.6 Hz, 1.8 Hz, 2H), 1.61-1.42 (m, 4H), 0,90 $(\mathrm{t}, J 6.9 \mathrm{~Hz}, 3 \mathrm{H}) ;{ }^{13} \mathrm{C} \mathrm{NMR}\left(\mathrm{CDCl}_{3}\right) \delta 131.7,128.6,128.4$,
$125.2,123.3,120.3,118.2,99.5,87.2,78.4,30.7,29.6$, 19.5, 13.6; LRMS $\mathrm{m} / \mathrm{z}$ (relative intensity) 208 (55), 178 (100), 165 (96), 139 (23), 115 (35), 91 (15), 63 (15); Elemental Analysis Calcd. for $\mathrm{C}_{16} \mathrm{H}_{16} \mathrm{C}, 92.26, \mathrm{H}, 7.74$; Found C, 92.09, H, 7.72\%.

Pentadec-(7Z)-ene-5,9-diyne (10h). ${ }^{17}$ Yield: $0.158 \mathrm{~g}$ (78\%); ${ }^{1} \mathrm{H} \mathrm{NMR}\left(\mathrm{CDCl}_{3}\right) \delta 5.72(\mathrm{~s}, 2 \mathrm{H}), 2.42-2.35(\mathrm{~m}, 4 \mathrm{H})$, $1.57-1.35(\mathrm{~m}, 10 \mathrm{H}) 0.91(\mathrm{~m}, 6 \mathrm{H}) ;{ }^{13} \mathrm{C} \mathrm{NMR}\left(\mathrm{CDCl}_{3}\right) \delta 118.9$, 97.9, 97.8, 78.3, 31.0, 30.7, 22.4, 22.2, 21.9, 19.7, 19.4, 13.9, 13.6; LRMS $m / z$ (relative intensity) 202 (34), 145 (26), 131 (68), 117 (87), 103 (32), 91 (100), 77 (37), 63 (15).

\section{Acknowledgements}

The authors acknowledge the following agencies for support: CNPq and FAPESP.

\section{References}

1. Dabdoub, M. J.; Dabdoub, V. B.; Comasseto, J. V.; Petragnani, N.; J. Organomet. Chem. 1986, 308, 211; Tucci, F. C.; Chieffi, A.; Comasseto, J. V.; Marino; J. P., J. Org. Chem. 1996, 61, 4975; Zeni, G.; Formiga, H. B.; Comasseto, J. V.; Tetrahedron Lett. 2000, 41, 1311; Marino, J. P.; Nguyen, H. N.; J. Org. Chem. 2002, 67, 6291; for a review see: Comasseto, J. V.; Barrientos-Astigarraga, R. E.; Aldrichimica Acta 2000, 33, 66; Vieira, M. L.; Zinn, F. K.; Comasseto, J. V.; J. Braz. Chem. Soc. 2001, 12, 586; for the synthesis of telluroenynes see: Dabdoub, M. J.; Dabdoub, V, B.; Comasseto, J. V.; Tetrahedron Lett. 1992, 33, 2261.

2. Minkin, V. J.; Sadekov, I. D.; Rivkin, B. B.; Zakharov, A. V.; Nivorozhkin, V. L.; Kompans, E.; Struchkov, V. T.; J. Organomet. Chem. 1997, 233, 536; Barrientos-Astigarraga, R. E.; Sumida, C. Y.; Castelani, P.; Comasseto, J. V.; Tetrahedron Lett. 1999, 40, 7717; Barrientos-Astigarraga, R. E.; Castelani, P.; Sumida, C. Y.; Zukerman-Schpector, J.; Comasseto, J. V.; Tetrahedron 2002, 58, 1051.

3. Moraes, D. N.; Barrientos-Astigarraga, R. E.; Castelani, P.; Comasseto, J. V.; Tetrahedron 2000, 56, 3327.

4. Krause, N., ed.; Modern Organocopper Chemistry, Wiley-VCH: Weinheim, 2002.

5. Marino, J. P.; McClure, M. S.; Holub, D. P.; Comasseto, J. V.; Tucci, F. C.; J. Am. Chem. Soc. 2002, 124, 1664.

6. Araujo, M. A.; Comasseto, J. V.; Synlett 1995, 1145.

7. Alexakis, A.; Cahiez, G.; Normant, J. F.; Synthesis 1979, 826.

8. Magriotis, P. A.; Scott, E. M.; Kim, K. D.; Tetrahedron Lett. 1991, 32, 6085.

9. Ghribi, A.; Alexakis, A.; Normant, J. F.; Tetrahedron Lett. 1984, 25, 3083; Rakotoarisoa, H.; Perez, R. G.; Mangeney, P.; Alexakis, A.; Organometallics 1996, 15, 1957. 
10. Border, D. B.; Doyle, T. D., eds.; Enediyne Antibiotics as Antitumor Agents, Mrcel Dekker: New York, 1995; Nicolaou, K. C.; Smith, A. L.; Acc. Chem. Res. 1992, 25, 497; Xi, Z.; Goldberg, I. H. In Comprehensive Natural Products Chemistry, Barton, D. H. R.; Nakanishi, K., eds.; Pergamon: Oxford, vol. 7, 1999.

11. Benites, P. J.; Rawat, D. S.; Zaleski, J. M.; J. Am. Chem. Soc. 2000, 122, 7208; Rawat, D. S.; Zaleski, J. M.; J. Am. Chem. Soc. 2001, 123, 9675.

12. Perrin, D. D.; Armarego, W. L. F.; Purification of Laboratory Chemicals, Pergamon Press: Great Britain, 1980.

13. Watson, S. C.; Eastmam, J. F.; J. Organomet. Chem. 1967, 9 , 165.
14. Bongiini, A.; Cardilho, G.; Orena, M.; Sandris, S.; Synthesis 1979, 618.

15. Brandsma, L.; Preparative Acetylenic Chemistry, $2^{\text {nd }}$ ed., Elsevier: Amsterdam, 1992.

16. Barros, S. M.; Dabdoub, M. J.; Dabdoub, V. M. B.; Comasseto, J. V.; Organometallics 1989, 8, 1661.

17. Melin, J.; Aparicio, F.; Galván, M.; Fuentealba, P.; Contreras, R.; J. Phys. Chem. A 2003, 107, 3831.

Received: October 7, 2003 Published on the web: April 30, 2004

FAPESP helped in meeting the publication costs of this article. 